\title{
Directed Therapy for Exfoliation Syndrome
}

\author{
Allison Angelilli ${ }^{1}$ and Robert Ritch ${ }^{*}, 1,2$ \\ ${ }^{I}$ Einhorn Clinical Research Center, New York Eye and Ear Infirmary, New York, NY, USA \\ ${ }^{2}$ Department of Ophthalmology, New York Medical College, Valhalla, NY, USA
}

\begin{abstract}
Exfoliation syndrome (XFS) is an age-related disorder of the extracellular matrix that leads the production of abnormal fibrillar material that leads to elevated intraocular pressure and a relatively severe glaucoma. Exfoliation material is deposited in numerous ocular tissues and extraocular organs. XFS is associated with ocular ischemia, cerebrovascular disease, neurodegenerative disease and cardiovascular disease. Current modalities of treatment include intraocular pressure lowering with topical antihypertensives, laser trabeculoplasty and filtration surgery. The disease paradigm for XFS should be expanded to include directed therapy designed specifically to target the underlying disease process. Potential targets include preventing the formation or promoting the depolymerization of exfoliation material. Novel therapies targeting trabecular meshwork may prove particularly useful in the care of exfoliative glaucoma. The systemic and ocular associations of XFS underscore the need for a comprehensive search for neuroprotective agents in its treatment.
\end{abstract}

\section{PATHOGENESIS AND PRESENTATION OF EXFOLIATION SYNDROME}

\section{Epidemiology and Clinical Presentation}

Exfoliation syndrome (XFS) is an age-related disease of the extracellular matrix characterized by the production and deposition of a fibrillar extracellular material in numerous ocular tissues. It is now recognized as the most common identifiable cause of open-angle glaucoma, affecting an estimated 60 to 70 million people worldwide $[1,2]$. Its reported prevalence rates vary extensively due to true population variations as well as the clinical criteria used and the ability of the examiner to detect the clinical signs of the disease.

The diagnosis of XFS is confirmed by the presence of white exfoliation material (XFM) at the pupillary border or on the anterior lens capsule in a characteristic distribution. A central disc is separated from a granular peripheral zone by an intermediate clear zone denuded of XFM by friction between the iris and anterior lens capsule during pupillary movement. The material on the anterior lens capsule causes rupture of iris pigment epithelial cells leading to pigment dispersion, trabecular meshwork hyperpigmentation, iris transillumination defects at the pupillary margin and loss of the pupillary ruff. Pigment dispersion after dilation can lead to marked IOP rise and may be one of the earliest manifestations of the disease.

Patients with XFS are twice as likely to convert from ocular hypertension to detectable glaucoma [3]. Exfoliative glaucoma (XFG) is more severe than primary open angle glaucoma (POAG). There is greater diurnal intraocular

*Address correspondence to this author at the Glaucoma Service, Department of Ophthalmology, The New York Eye and Ear Infirmary, 310 East 14th Street, New York, NY 10003, USA; Tel: (212) 673-5140; Fax: (212) 4208743; E-mail: ritchmd@earthlink.net pressure (IOP) fluctuation, greater visual field loss and optic nerve head damage at presentation, poorer response to medications, faster visual field progression and increased need for surgical intervention. At any given IOP, eyes with XFS are at significantly greater risk for glaucomatous damage, suggesting that pressure-independent factors play a significant role in visual field loss in these patients.

The prevalence of XFS increases with age in all affected populations. Approximately two-thirds of patients have unilateral disease on clinical examination; however, XFS is often detectable in the contralateral eye with conjunctival biopsy [4]. This finding supports the hypothesis that the development of XFS is similar to uveitis in that an underlying predisposition plus an environmental or immunologic trigger leads to disease manifestation.

\section{Pathogenesis of XFS}

The pathogenesis of XFS is defined by the accumulation of an abnormal fibrillar material biochemically related to the composition of the extracellular matrix that results either from excessive production and/or insufficient breakdown. The product has a unique structure as seen by electron microscopy [5]. Streeten et al. first hypothesized that XFS is an elastosis based on similarities of XFM and zonular fibers [6]. These characteristic fibrils, composed of microfibrillar subunits surrounded by an amorphous matrix, contain epitopes of elastic fibers such as fibrillin-1, elastin, tropoelastin, amyloid $\mathrm{P}$ and microfibril-associated glycoprotein, vitronectin and the cross-linking enzyme lysyloxidase $[7,8]$.

Two common single nucleotide polymorphisms (SNPs) in the coding region of the lysyl-oxidase-like 1 (LOXL1) gene on chromosome 15 have been identified and account for virtually all cases of XFS in several populations [9-12]. While the SNPs confer susceptibility to XFS, not all people with the SNPs will develop the disease. 
LOXL1 is a member of the lysyl oxidase enzyme family responsible for formation, stabilization and remodeling of elastic fibers. They are important for cross-linking of elastin, for providing a scaffold that ensures spatially defined deposition of elastin and for regulating the production of elastin $[13,14]$. The currently proposed pathogenesis of XFS is a stress-related, excessive production by elastogenic cells of elastic microfibrils that aggregate into a typical configuration via an enzymatic cross-linking process [15]. There is evidence that derangement of the balance of matrix metalloproteinases and their tissue inhibitors, increased oxidative stress and an impaired stress response contribute to impaired degradation and accumulation of fibers [16-19]. These fibers impair trabecular meshwork outflow resulting in an increased rise of IOP.

\section{Ocular and Systemic Associations}

XFS is associated with both chronic open-angle glaucoma and angle closure. The zonules and ciliary body are often diffusely coated with XFM and zonules are often frayed and broken. Zonular fragility and poor pupillary dilation contribute to an increased incidence of serious complications during cataract extraction, including zonular dehiscence and vitreous loss. Phacodonesis and even subluxation of the lens or intraocular lens is common.

It appears that XFS is etiologically related to cataract formation itself. Decreased aqueous humor ascorbic acid concentrations in XFS [20], increased malondialdehyde concentrations [21] and increased 8-iso-prostaglandin $\mathrm{F}_{2 \mathrm{a}}$ [22] suggest that free radicals and oxidative damage play a role in the disease process. There is also breakdown of the bloodaqueous barrier with resultant increased protein concentrations in the anterior chamber.

Ocular ischemia is clearly correlated with XFS. An increased frequency of retinal vein occlusion has been noted in XFS [23-25] and iris hypoperfusion can be detected angiographically and histopathologically [26, 27]. Patchy iris neovascularization can be seen when capillary dropout is extensive $[7,15]$. Deposits of XFM can be found in the walls of vortex veins and central retinal veins. Not surprisingly, cardiovascular and cerebrovascular disorders are emerging as systemic associations of XFS. XFM has been found electron microscopically in the heart, liver, lungs, kidney and meninges in patients with XFS $[28,29]$. Ocular, retrobulbar, cerebral and carotid blood flow are reduced [30-34]. XFS is a risk factor for cardiovascular disease [35] and several reports have linked XFS with Alzheimer's disease, hearing loss and transient ischemic attacks [36-39]. The pathologic connection between XFM and the functional deficits seen is currently unclear.

\section{DIRECTED THERAPY FOR XFS}

As the term implies, directed therapy aims to specifically address and interfere with the underlying disease pathogenesis. Ophthalmologists have begun to recognize the inadequacy of therapy limited solely to IOP reduction, particularly with the significant potential morbidities associated with XFS. The widespread systemic and ocular manifestations of XFS underscore the need for a broad and comprehensive treatment paradigm. It is essential that future investigations focus on disease-specific therapeutic modalities.

\section{Directed Ocular Antihypertensive Therapy}

The management of XFS currently hinges on ocular antihypertensive therapy and initially can be achieved with topical therapy in many cases. Prostaglandin analogues such as latanoprost and travoprost reduce IOP at each time point in the 24-hour diurnal curve as compared to untreated IOP, with travoprost conferring a slightly higher level of reduction [40]. Latanoprost reduced IOP and narrowed the range of diurnal fluctuation in comparison to timolol in XFS [41].

Pilocarpine has multiple beneficial actions in eyes with XFS. Not only does it lower IOP, but by increasing aqueous outflow, it should enable the trabecular meshwork to clear more rapidly, and by limiting pupillary movement, should slow disease progression. Theoretically, miotics should be the first line of treatment. Prostaglandin analogues are complementary in increasing uveoscleral outflow facility. By decreasing outflow, aqueous suppressants may cause longterm worsening of trabecular function that may be particularly detrimental in XFS [42].

Unfortunately, pilocarpine is underutilized in the treatment of glaucoma due to the possibility of decreased vision or the misconception that it must be used q.i.d. It is our experience that pilocarpine $2 \%$ q.h.s. can provide significant limitation of pupillary movement without the inconvenience of q.i.d. dosing and dimming of vision. Pilocarpine can also blunt an early morning IOP spike [43].

Argon laser trabeculoplasty is particularly effective in XFS [44]. Primary ALT can delay the need for topical therapy in some patients, although there is a general decrease in efficacy over time, with $35-55 \%$ remaining medication free at 3-5 years [45]. A minority of patients will experience a sudden and dramatic rise in IOP within two years following treatment, presumably due to continued pigment liberation overwhelming the meshwork. Pilocarpine 2\% q.h.s. may provide protection from this. Limited data on selective laser trabeculoplasty (SLT) suggests that success may be comparable, although prospective studies are necessary to confirm this and also to compare results with argon laser trabeculoplasty [46]. Marked IOP elevations associated with pigment release in patients with heavily pigmented trabecular meshworks necessitating filtration surgery may occur after SLT [47]. Trabeculectomy with antifibrotic therapy leads to comparable IOP reduction in XFS and OAG, and offers greater 24-hour IOP control than medical therapy alone [48].

The investigation of surgical therapy directed at trabecular dysfunction is particularly sensible for XFS. Trabeculotomy has been reported as successful in XFS although additional trials are required [49-51]. Goniotomy is also successful (Kim JH, Sbeity Z, Ritch R: Long-term results of goniotomy and cataract surgery for exfoliative glaucoma. Southeast Asian Glaucoma Interest Group, Seoul, Republic of Korea, Sept. 25, 2008.) Jacobi and Krieglstein [52] described a procedure in which trabecular aspiration improves outflow facility.

\section{Novel Therapies Targeting Trabecular Meshwork}

Drugs that alter the structure of the trabecular meshwork may be particularly effective in XFS. Latrunculin B is currently being evaluated in human trials for its ability to 
bind to free actin in trabecular cells leading, to temporary disintegration of the existing actin cytoskeletin thereby increasing aqueous outflow [53, 54]. Unlike previous compounds with similar mechanisms of action such as sodium-EDTA and cytochalasin B [55, 56], Latrunculin has no known adverse effects on retinal and corneal function [57-59]. Ideally, latrunculin would be effective as a depot medication with once a week or once a month dosing.

\section{Treatment Directed at XFM}

Future therapy of XFS should be directed at preventing formation of XFM or depolymerizing existing XFM. An intriguing line of research is an investigation of the factors that protect the contralateral eye from developing XFS. If the development of the disease in the second eye is actively suppressed, as by an immune mechanism, then elucidation of this could lead to a novel treatment. The systemic ramifications of XFS and the morbidity associated with these diseases highlight the need for a therapy that prevents aggregation of XFM microfibrils disaggregating the microfibrils.

Homocysteine is an amino acid, elevations of which result from a disturbance of methionine metabolism. Hyperhomocysteinemia is a widely recognized cardiovascular risk factor and is reversible with dietary supplementation with folic acid, pyridoxine (vitamin B6) and cyanocobalamine (vitamin B12) [60, 61]. Hyperhomocysteinemia is associated with disruption of the elastic fibers of the extracellular matrix leading to vascular disease [62]. Elevated homocysteine levels are present in blood, aqueous and tears of patients with XFS, and there are decreased serum levels of cyanocobalamin, pyridoxine and folic acid [63-66]. The concept of a relationship between hyperhomocysteinemia is supported by the propensity for cardiovascular and cerebrovascular disease in patients with XFS. By the same virtue, it is possible that treatment of hyperhomocysteinemia could be beneficial in XFS as it is in cardiovascular disease.

Folic acid deficiency leads to altered expression of genes involved in cell signaling, the cytoskeleton and the extracellular matrix [67]. Actin disrupting agents, such as latrunculin $\mathrm{B}$, reversibly increase the proportion of receptors on the cell surface and increase [68] the rate of 5methyltetrahydrofolate delivery [69]. Pyridoxine is an essential micronutrient involved in a variety of critical metabolic reactions including carbohydrate metabolism, sphingolipid biosynthesis and degradation, amino acid metabolism (including that of homocysteine), and neurotransmitter metabolism [70]. Therefore, deficiency of this essential micronutrient in humans leads to a variety of adverse conditions and to disturbances in normal cellular metabolism. Pyridoxine also plays a role in the integrity of the extracellular matrix [71]. Cyanocobalamin is important for the normal functioning of the brain and nervous system and for the formation of blood. It is normally involved in the metabolism of every cell of the body, especially affecting the DNA synthesis and regulation but also fatty acid synthesis and energy production.

The discovery of the role of LOXL1 polymorphisms in XFS draws attention towards lysyl oxidase and its role in XFM production. Lysyl oxidase is inhibited by homocysteine in vascular endothelia and is also regulated by transforming growth factor- $\beta 1$ (TGF- $\beta 1$ ) [72, 14]. Levels of TGF- $\beta 1$ are significantly increased in the aqueous humor of XFS and TGF- $\beta 1$ may be responsible for overproduction of extracellular matrix in XFS [73]. Further information on the biochemical pathway of the production of XFM is critical if we hope to modify the treatment of XFS significantly.

\section{Treatment Targeting Non-pressure Dependent Mechanisms}

Patients with XFS have an increased likelihood to have coexisting cerebrovascular and cardiovascular disease, and systemic agents that provide neuroprotection and vasoprotection may prove to be an important therapeutic tactic in the treatment of XFS. Pharmacologic treatment of non-IOP dependent mechanisms in glaucoma has traditionally been limited to calcium channel blockers that are widely used in treatment of hypertension, coronary artery disease, migraines and Raynaud's disease. The effect of calcium channel blockers on visual field progression and ocular blood flow is disputed [74-76].

Gingko biloba extract (GBE) has been used in Chinese medicine since $3000 \mathrm{BC}$ for the treatment of aging, dementia, tinnitus, bronchoconstriction and sexual dysfunction with minimal side effects. It improves peripheral, cerebral and ocular blood flow, prevents platelet aggregation and protects against free radical damage [77, 78]. Neurons in tissue culture are protected from toxicityinduced apoptosis with GBE, and there is mixed evidence that GBE improves function in patients with Alzheimer's disease and vascular dementia $[79,80]$. Further elucidation by prospective trials of GBE in glaucoma is warranted.

Curcumin is an anti-oxidant extracted from the plant Curcuma longa and is a component of the commonly used Indian spice turmeric. Curcumin has antioxidant, antiinflammatory, anti-angiogenic and anti-neoplastic activity via the inhibition of numerous mediators of inflammation [81-85]. Clinical trials for the treatment of gastrointestinal and other neoplasms as well as Alzheimer's disease are under way. Common therapeutic doses cause only mild gastrointestinal discomfort in a minority of patients.

Resveratrol is a compound found in the skin of red grapes. It is an effective antioxidant and protects against degeneration of neurons in ischemia. It is hypothesized that resveratrol is responsible for the "French paradox", the observation that the French have a lower incidence of heart disease despite a high fat diet due to the consumption of red wine. It is an effective antioxidant and prevents neuron degeneration $[84,85]$.

\section{CONCLUSION}

XFS is a systemic disorder with components of lowgrade inflammation and oxidative damage leading to accumulation of XFM. The ocular morbidity associated with XFS far exceeds that of open-angle glaucoma, and a host of systemic diseases with considerable morbidity and mortality are associated with XFS. Further elucidation of the genetics and pathophysiology of XFS is crucial to our ability to treat this disease effectively. There is a role for non-IOP lowering treatment modalities in XFS as there is significant evidence of generalized ischemia and neurodegeneration. With this 
directed investigation, there is hope that XFS will be both a preventable and curable disease.

\section{ACKNOWLEDGEMENT}

Supported in part by the Leo and Mary Birenbaum Research Fund of the New York Glaucoma Research Institute, New York, NY, USA.

\section{REFERENCES}

[1] Ritch R. Exfoliation syndrome: the most common identifiable cause of open angle glaucoma. J Glaucoma 1994; 3: 176-8.

[2] Ritch R, Schlötzer-Schrehardt U, Konstas A. Why is glaucoma associated with exfoliation syndrome? Prog Retinal Eye Res 2003; 22: $253-75$.

[3] Leske MC, Heijl A, Hussein, et al. Factors for glaucoma progression and the effect of treatment. The early manifest glaucoma trial. Arch Ophthalmol 2003; 121: 48-56.

[4] Prince AM, Streeten BW, Ritch R, Dark A, Sperling M. Preclinical diagnosis of pseudoexfoliation syndrome. Arch Ophthalmol 1987; 105: 1076-82.

[5] Naumann G, Schlötzer-Schrehardt U, Küchle M. Pseudoexfoliation syndome for the comprehensive ophthalmologist: Intraocular and systemic manifestations. Ophthalmology 1998; 105: 951-68.

[6] Streeten B, Gibson S, Dark A. Pseudoexfoliation material contains an elastic microfibrillar-associated glycoprotein. Trans Am Ophthalmol Soc 1986; 84: 304-20.

[7] Ritch R, Schlötzer-Schrehardt U. Exfoliation syndrome. Surv Ophthalmol 2001; 45: 265-315.

[8] Ovodenko B, Rostagno T, Neubert T, et al. Proteomic analysis of lenticular exfoliation deposits. Invest Ophthalmol Vis Sci 2007; 48: 1447-57.

[9] Thorliefsson G, Magnusson K, Sulem P, et al. Common sequence variants in the LOXL1 gene confer susceptibility to exfoliation glaucoma. Science 2007; 317: 1397-400.

[10] Fingert J, Alward W, Kwon Y, et al. LOXL1 mutations are associated with exfoliation syndrome in patients from the midwestern United States. Am J Ophthalmol 2007; 144: 974-75.

[11] Hewitt A, Sharma S, Burdon K, et al. Ancestral LOXL1 variants are associated with pseudoexfoliation in Caucasian Australians but with markedly lower penetrance than in Nordic people. Hum Mol Genet 2008; 17: 710-16.

[12] Hayashi H, Gotoh N, Ueda Y, et al. Lysyl Oxidase-like 1 polymorphisms and exfoliation syndrome in the Japanese population. Am J Ophthalmol 2008; 145: 582-85.

[13] Liu X, Zhao Y, Gao J, et al. Elastic fiber homeostasis requires lysyl oxidase-like 1 protein. Nat Genet 2004; 36: 178-82.

[14] Oleggini R, Gastaldo N, Di Donato A. Regulation of elastin promoter by lysyl oxidase and growth factors: cross control of lysyl oxidase on TGF-beta1 effects. Matrix Biol 2007; 26: 494-505.

[15] Schlötzer-Schrehardt U, Naumann G. Ocular and systemic pseudoexfoliation syndrome. Am J Ophthalmol 2006; 141: 921-37.

[16] Schlötzer-Schrehardt U, Lommatzsch, Küchle M, et al. Matrix metalloproteinases and their inhibitors in aqueous humor of patients with pseudoexfoliation syndrome/glaucoma and primary open-angle glaucoma. Invest Ophthalmol Vis Sci 2003; 44: 111725.

[17] Ho S, Dogar G, Wang J, et al. Elevated aqueous humor tissue inhibitor of matrix metalloproteinase- 1 and connective tissue growth factor in pseudoexfoliation syndrome. $\mathrm{Br} \mathrm{J}$ Ophthalmol 2008; 89: 169-73.

[18] Rönkkö S, Rekonen P, Kaarniranta K, et al. Matrix metalloproteinases and their inhibitors in the chamber angle of normal eyes and patients with primary open-angle glaucoma and exfoliation glaucoma. Graefes Arch Clin Exp Ophthalmol 2007; 245: 697-704

[19] Koliakos G, Schlötzer-Schrehardt U, Konstas A, et al. Transforming and insulin like growth factor in the aqueous humor of patients with exfoliation syndrome. Graefes Arch Clin Exp Ophthalmol 2001; 239: 482-7.

[20] Koliakos G, Konstas A, Schlötzer-Schrehardt U, et al. Ascorbic acid concentration is reduced in the aqueous humor of patients with exfoliation syndrome. Am J Ophthalmol 2002; 134: 879-83.
[21] Yilmaz A, Adiguzel U, Tamer L, et al. Serum oxidant/antioxidant balance in exfoliation syndrome. Clin Exp Ophthalmol 2005; 33: 63-6.

[22] Koliakos G, Konstas A, Schlötzer-Schrehardt U, et al. 8-Isoprostaglandin F2a and ascorbic acid concentration in the aqueous humor of patients with exfoliation syndrome. Br J Ophthalmol 2003; 87: 353-6.

[23] Saatci OA, Ferliel S, Ferliel M, et al. Pseudoexfoliation and glaucoma in eyes with retinal vein occlusion. Int Ophthalmol 1999; 23: $75-8$.

[24] Cursiefen C, Hammer T, Küchle M, et al. Pseudoexfoliation syndrome in eyes with ischemic central retinal vein occlusion. A histopathologic and electron micrographic study. Acta Ophthalmol Scand 2001; 79: 476-8.

[25] Ritch R, Prata TS, de Moraes CGV, et al. Association of exfoliation syndrome and central retinal vein occlusion: an ultrastructural analysis. Acta Ophthalmol Scand 2008; 86: 565-68.

[26] Laatkainen L. Fluorescein angiographic studies of the peripapillary and perilimbal regions in simple, capsular and low-tension glaucoma. Acta Ophthalmol 1971; 3: 3-83.

[27] Hammer T, Schlötzer-Schrehardt U, Naumann GOH. Unilateral or asymmetric pseudoexfoliation syndrome? An ultrastructural study. Arch Ophthalmol 2001; 119: 1023-31.

[28] Schlötzer-Schrehardt U, Koca MR, Naumann GOH, Volkholz H. Pseudoexfoliation syndrome. Ocular manifestation of a systemic disorder? Arch Ophthalmol 1992; 110: 1752-56.

[29] Streeten B, Li Z, Wallace R, et al. Pseudoexfoliative fibrillopathy in visceral organs of a patient with pseudoexfoliation syndrome. Arch Ophthalmol 1992; 110: 1757-62.

[30] Yüksel N, Anik Y, Kilic A, et al. Cerebrovascular blood flow velocities in pseudoexfoliation. Graefes Arch Clin Experiment Ophthalmol 2006; 244: 316-21.

[31] Yüksel N, Karabas V, Arslan A, et al. Ocular hemodynamics in pseudoexfoliation syndrome and pseudoexfoliation glaucoma Ophthalmology 2001; 108: 1043-49.

[32] Scullica L, Buceti R, Castagna I, et al. Functional aspects of pseudoexfoliation: Physiopathologic features. New Trends Ophthalmol 1993; 8: 163-8.

[33] Sibour G, Finazzo C, Boles Carenini A. Monolateral pseudoexfoliatio capsulae: A study of choroidal blood flow. Acta Opthalmol Scand 1997; 75(Suppl 224): 13-4.

[34] Repo L, Suhonen M, Teräsvirta M, Koivisto J. Color Doppler imaging of the ophthalmic artery blood flow spectra of patients who have had a transient ischemic attack. Ophthalmology 1995 102: $1199-205$

[35] Andrikopoulos G, Mela E, Georgakopoulos, et al. Pseudoexfoliation syndrome prevalence in Greek patients with cataract and its association to glaucoma and coronary artery disease. Eye 2009; 23 : 442-47.

[36] Hagadus R, Wandel T, Ritch R, et al. Pseudoexfoliation in patients with Alzheimer's disease. Invest Ophthalmol Vis Sci 1989; 30(Suppl): 27.

[37] Linnér E, Popovic C, Gottfries C, et al. The exfoliation syndrome in cognitive impairment of cerebrovascular or Alzheimer's type. Acta Opthalmol Scand 2001; 79: 283-85.

[38] Shaban R, Asfour W. Ocular pseudoexfoliation associated with hearing loss. Saudi Med J 2004; 25: 1254-57.

[39] Mitchell P, Wang J, Smith W. Association of pseudoexfoliation with increased vascular risk. Am J Ophthalmol 1997; 124: 685-7.

[40] Konstas A, Kozobolis V, Katsimpris I, et al. Efficacy and safety of latanoprost versus travoprost in exfoliative glaucoma patients. Ophthalmol 2007; 114: 653-7.

[41] Konstas A, Holló M, Irkec M, et al. Diurnal IOP control with bimatoprost $v s$ latanoprost in exfoliative glaucoma: a crossover observer-masked 3-center study. Br J Ophthalmol 2007; 91: 75760 .

[42] Becker B. Does hyposecretion of aqueous humor damage the trabecular meshwork? J Glaucoma 1995; 4: 303-5.

[43] Barkana Y, Anis S, Liebmann J, et al. Intraocular pressure monitoring outside of normal office hours in patients with glaucoma is clinically useful. Arch Ophthalmol 2006; 124: 793-9.

[44] Ritch R, Podos S. Laser trabeculoplasty in exfoliation syndrome. Bull NY Acad Med 1983; 59: 339-44.

[45] Odberg T, Sandvik L. The medium and long-term efficacy of primary argon laser trabeculoplasty in avoiding topical medication in open-angle glaucoma. Acta Ophthalmol 1999; 77: 176-81. 
[46] Gracner T. Intraocular pressure response of capsular glaucoma and primary open-angle glaucoma to selective $\mathrm{Nd}$ :YAG laser trabeculoplasty: A prospective, comparative clinical trial. Eur J Ophthalmol 2008; 12: 287-92.

[47] Harasymowycz P, Papamatheakis D, Latina M, et al. Selective Laser Trabeculoplasty (SLT) complicated by intraocular pressure elevation in eyes with heavily pigmented trabecular meshworks. Am J Ophthalmol 2005; 139: 1110-3.

[48] Konstas A, Topouzis F, Leliopoulou O, et al. 24-hour intraocular pressure control with maximum medical therapy compared with surgery in patients with advanced open-angle glaucoma. Ophthalmol 2006; 113: 761-5

[49] Gillies WE. Trabeculotomy in pseudoexfoliation of the lens capsule. Br J Ophthalmol 1977; 61: 297-305.

[50] Tanihara $\mathrm{H}$, Negi A, Akimoto $\mathrm{M}$, et al. Surgical effect of trabeculotomy ab externo on adult eyes with primary open-angle glaucoma and pseudoexfoliation syndrome. Arch Ophthalmol 1993; 111: 1653-61.

[51] Honjo $\mathrm{M}$, Tanihara $\mathrm{H}$, Inatani $\mathrm{M}$, et al. Phacoemulsification, intraocular lens implantation and trabeculotomy to treat pseudoexfoliation syndrome. J Cataract Refract Surg 1998; 24: 781-6.

[52] Jacobi PC, Dietlein T, Krieglstein G. Bimanual trabecular aspiration in pseudoexfoliation glaucoma- an alternative in nonfiltering glaucoma surgery. Ophthalmology 1998; 105: 886-94.

[53] Peterson J, Tian B, Geiger B, Kaufman P. Effect of latrunculin-B on outflow facility in monkeys. Exp Eye Res 2000; 70: 307-14.

[54] Ethier C, Read A, Chan D. Effects of latrunculin-B on outflow facility and trabecular meshwork structure in human eyes. Invest Ophthalmol Vis Sci 2006; 47: 1991-8.

[55] Kaufman P, Bárány E. Cytochalasin B reversibly increases outflow facility in the eye of the cynomolgus monkey. Invest Ophthalmol Vis Sci 1977; 16: 47-53.

[56] Bill A, Lütjen-Drecoll E, Svedbergh B. Effects of intracameral sodium-EDTA and EGTA on aqueous outflow routes in the monkey eye. Invest Ophthalmol Vis Sci 1980; 19: 492-504.

[57] Kiland J, Miller C, Kim C, et al. Effect of H-7 and Lat-B on retinal physiology. Curr Eye Res 2006; 31: 441-55.

[58] Okka M, Tian B, Kaufman P. Effect of low-dose latrunculin-B on anterior segment physiologic features in the monkey. Arch Ophthalmol 2004; 122: 1482-8.

[59] Sabanay I, Tian B, Gabelt B, et al. Latrunculin-B effects on trabecular meshwork and corneal endothelial morphology in monkeys. Exp Eye Res 2006; 82: 236-46.

[60] Selhub J. The many facets of hyperhomocysteinemia: studies from the Framingham cohorts. J Nutr 2006; 136(6 Suppl): 1726S-30S

[61] Lobo A, Naso A, Arheart K, et al. Reduction of homocysteine levels in coronary artery disease by low-dose folic acid combined with vitamins B6 and B12. Am J Cardiol 1999; 83: 821-5.

[62] Starcher B, Hill C. Elastin defects in the lungs of avian and murine models of homocysteinemia. Exp Lung Res 2005; 31: 873-85.

[63] Leibovitch I, Kurtz S, Shemesh G, et al. Hyperhomocysteinemia in pseudoexfoliation glaucoma. J Glaucoma 2003; 12: 36-9.

[64] Vessani R, Liebmann J, Jofe M, Ritch R. Plasma homocysteine is elevated in patients with exfoliation syndrome. Am J Ophthalmol 2003; 136: 41-6.

[65] Bleich S, Roedl J, Von Ahsen N, et al. Elevated homocysteine levels in aqueous humor of patients with pseudoexfoliation glaucoma. Am J Ophthalmol 2004; 138: 162-4.

[66] Roedl J, Bleich S, Reulbach U, et al. Homocysteine in tear fluid of patients with pseudoexfoliation glaucoma. J Glaucoma 2007; 16: 234-9.
[67] Katula K, Heinloth A, Paules R. Folate deficiency in normal human fibroblasts leads to altered expression of genes primarily linked to cell signaling, the cytoskeleton and extracellular matrix. J Nutr Biochem 2007; 18: 541-52.

[68] Holven K, Halvorsen B, Schulz H, et al. Increased levels of Creactive protein and interleukin-6 in hyperhomocysteinemic subjects. Scand J Clin Lab Invest 2006; 66: 45-54.

[69] Lewis C, Smith A, Kamen B. Receptor-mediated folate uptake is positively regulated by disruption of the actin cytosckeleton. Cancer Res 1998; 15: 2952-6.

[70] Merrill A, Henderson M. Diseases associate with defects in vitamin B6 metabolism or utilization. Ann Rev Nutr 1987; 7: 137-56.

[71] Massé P, Yamauchi M, Mahuren J, et al. Connective tissue integrity is lost in vitamin B6-deficient chicks. J Nutr 1995; 125 26-34.

[72] Raposo B, Rodriguez C, Martínez-Gonzáles, BL. High levels of homocysteine inhibit lysyl oxidase (LOX) and downregulate LOX expression in vascular endothelial cells. Atherosclerosis 2004; 177 : $1-8$.

[73] Schlötzer-Schrehardt U, Küchle M, Rummelt C, Naumann G. Role of transforming growth factor- $\beta$ and its latent form binding protein in pseudoexfoliation syndrome. Invest Ophthalmol Vis Sci 1999; 40: $\mathrm{S} 278$

[74] Gasser P, Flammer J, Guthauser U, et al. Short- and long-term effect of nifedipine on the visual field in patients with presumed vasospasm. J Int Med Res 1990; 18: 334.

[75] Kitazawa Y, Shirai H, Go F. The effect of calcium antagonist on visual field in low-tension glaucoma. Graefes Arch Clin Exp Ophthalmol 1989; 227: 408-12.

[76] Leighton D, Phillips C. Systemic blood pressure in open-angle, low-tension glaucoma, and the normal eye. Br J Ophthalmol 1972; 52: 447-59.

[77] Fitzl G, Welt K, Wassilew G, et al. The influence of hypoxia on the myocardium of experimentally diabetic rats with and without Gingko biloba extract. Exp Toxicol Pathol 2001; 52(6): 557-68.

[78] Sastre J, Lloret A, Borras C, et al. GBE EGb 761 protects against mitochondrial aging in the brain and in the liver. Cell Mol Biol 2002; 48: 685-92.

[79] Ahlemeyer B, Mowes A, Krieglstein J. Inhibition of serum deprivation- and staurosporine-imduced neuronal apoptosis by Gingko biloba extract and some of its constituents. Eur J Pharmacol 1999; 367: 423-30.

[80] Le Bars P, Katz M, Berman N, et al. A placebo-controlled, doubleblind, randomized trial of an extract of Gingko biloba for dementia. JAMA 1997; 278: 1327-32.

[81] Weber W, Hunsaker L, Abcouwer S, et al. Anti-oxidant activities of curcumin and related enones. Bioorg Med Chem 2005; 13 : 3811-20.

[82] Wang Q, Sun A, Simonyi A, et al. Neuroprotective mechanisms of curcumin against cerebral ischemia-induced neuronal apoptosis and behavioral deficits. J Neurosci Res 2005; 82: 138-48.

[83] Zbarsky V, Datla K, Parkar S, et al. Neuroprotective properties of the natural phenolic antioxidants curcumin and naringenin but not quercitin and fisetin in a 6-OHDA model of Parkinson's disease. Free Radic Res 2005; 39: 1119-25.

[84] Shigematsu S, Ishida S, Hara M, et al. Resveratrol, a red wine constituent polyphenol, prevents superoxide-dependent inflammatory responses induced by ischemia/reperfusion, plateletactivating factor, or oxidants. Free Radic Biol Med 2003; 34: 81017.

[85] Araki T, Sasaki Y, Milbrandt J. Increased nuclear NAD biosynthesis and SIRT1 activation prevent axonal degeneration. Science 2004; 305: 954-55.

This is an open access article licensed under the terms of the Creative Commons Attribution Non-Commercial License (http: //creativecommons.org/licenses/by$\mathrm{nc} / 3.0 /$ ) which permits unrestricted, non-commercial use, distribution and reproduction in any medium, provided the work is properly cited. 